\title{
Síndrome de Dificuldade Respiratória Aguda: Casuística de Dois Anos numa Unidade de Cuidados Intensivos
}

\author{
Acute Respiratory Distress Syndrome: Case Series, Two Years at an Intensive \\ Care Unit
}

\author{
Lúcia TABORDA ${ }^{1}$, Filipa BARROS², Vitor FONSECA², Manuel IRIMIA², Ramiro CARVALHO ${ }^{2}$, Cláudia DIOGO², Armindo \\ RAMOS $^{2}$ \\ Acta Med Port 2014 Mar-Apr;27(2):211-217
}

\section{RESUMO}

Introdução: A Síndrome de Dificuldade Respiratória Aguda apresenta incidência e mortalidade significativas em Cuidados Intensivos, justificando estudos adicionais, nomeadamente para definição de novas abordagens terapêuticas. Os autores propuseram-se caracterizar os casos duma Unidade de Cuidados Intensivos em dois anos.

Material e Métodos: Procedeu-se a um estudo observacional retrospectivo dos casos admitidos numa Unidade de Cuidados Intensivos, cumprindo os critérios diagnósticos da American-European Consensus Conference on ARDS, tendo sido excluídos os não ventilados invasivamente. Pesquisados e submetidos a tratamento estatístico: dados demográficos, etiologia do Síndrome de Dificuldade Respiratória Aguda, comorbilidades, Índices de Gravidade, PaO2/FiO2, modalidades e parâmetros ventilatórios, compliance pulmonar, dias de ventilação mecânica invasiva, corticoterapia, terapêuticas de resgate, complicações, duração do internamento, óbitos.

Resultados: Obtiveram-se 40 doentes, com uma mediana de 72,5 anos (amplitude interquartil 22) e um ratio feminino:masculino $\approx 1: 1,86$. Cinquenta e cinco por cento dos Síndrome de Dificuldade Respiratória Aguda tiveram etiologia pulmonar. A média do PaO2/ FiO2 mínimo foi 88mm Hg (IC 95\%: 78,5-97,6). A média da PEEP máxima aplicada foi 12,4 cmH2O (Desvio Padrão 4,12) e a média do Volume Corrente máximo utilizado foi $8,2 \mathrm{~mL} / \mathrm{Kg}$ peso ideal (IC 95\%: 7,7-8,6). A mediana dos dias de ventilação mecânica invasiva foi 10 . Em $47,5 \%$ dos doentes foram administrados corticóides. Em 52,5\% foi executado recrutamento alveolar. A complicação mais frequente foi a Pneumonia Associada a Ventilação (20\%). A mediana da duração do internamento foi 10,7 dias (amplitude interquartil 10,85). Faleceram $60 \%$ dos doentes. A probabilidade de outcome favorável 'não óbito na Unidade de Cuidados Intensivos' foi $4,4 x$ superior nos doentes sob corticoterapia e 11x superior nos doentes com idade < 65 anos.

Discussão e Conclusões: A Síndrome de Dificuldade Respiratória Aguda associa-se a internamentos prolongados e significativa mortalidade. Novos estudos prospectivos serão necessários para confirmar o benefício dos corticóides, bem como identificar o/(s) subgrupo/(s) de doentes que mais justificam a sua utilização.

Palavras-chave: Síndrome de Dificuldade Respiratória no Adulto; Ventilação Mecânica; Corticosteroides; Prognóstico; Portugal.

\section{ABSTRACT}

Introduction: Acute Respiratory Distress Syndrome has a significant incidence and mortality at Intensive Care Units. Therefore, more studies are necessary in order to develop new effective therapeutic strategies. The authors have proposed themselves to characterize Acute Respiratory Distress Syndrome patients admitted to an Intensive Care Unit for 2 years.

Material and Methods: This was an observational retrospective study of the patients filling the Acute Respiratory Distress Syndrome criteria from the American-European Consensus Conference on ARDS, being excluded those non invasively ventilated. Demographic data, Acute Respiratory Distress Syndrome etiology, comorbidities, Gravity Indices, PaO2/FiO2, ventilator modalities and programmation, pulmonary compliance, days of invasive mechanical ventilation, corticosteroids use, rescue therapies, complications, days at Intensive Care Unit and obits were searched for and were submitted to statistic description and analysis.

Results: A 40 patients sample was obtained, with a median age of 72.5 years (interquartile range $=22$ ) and a female:male ratio of $\approx 1: 1.86$. Fifty five percent of the Acute Respiratory Distress Syndrome cases had pulmonary etiology. The mean minimal PaO2/FiO2 was $88 \mathrm{mmHg}(\mathrm{Cl} 95 \%$ : 78.5-97.6). The mean maximal applied PEEP was $12.4 \mathrm{cmH} 2 \mathrm{O}$ (Standard Deviation 4.12) and the mean maximal used tidal volume was $8.2 \mathrm{~mL} / \mathrm{Kg}$ ideal body weight (Cl 95\%: 7.7-8.6). The median invasive mechanical ventilation days was 10 . Forty seven and one half percent of the patients had been administered corticosteroids and $52.5 \%$ had been submitted to recruitment maneuvers. The most frequent complication was Ventilator Associated Pneumonia (20\%). The median Intensive Care Unit stay was 10.7 days (interquartile range 10.85). The fatality rate was $60 \%$. The probability of the favorable outcome 'non-death in Intensive Care Unit' was $4.4 \mathrm{x}$ superior for patients who were administered corticosteroids and $11 \mathrm{x}$ superior for patients < 65 years old.

Discussion and Conclusions: Acute Respiratory Distress Syndrome is associated with long hospitalization and significant mortality. New prospective studies will be necessary to endorse the potential benefit of steroid therapy and to identify the subgroups of patients that warrant its use.

Keywords: Adrenal Cortex Hormones; Critical Care; Prognosis; Respiratory Distress Syndrome, Adult; Respiration, Artificial.

\section{INTRODUÇÃO}

A Síndrome de Dificuldade Respiratória Aguda, com o acrónimo ARDS remete, a nível internacional, para uma síndrome clínica fisiopatologicamente caracterizada por lesão pulmonar aguda, nomeadamente de etiologia primaria- mente pulmonar (em contexto de pneumonia ou aspiração de conteúdo gástrico, entre outros, condicionando danos do epitélio alveolar) ou extra-pulmonar (secundária por exemplo a sépsis e/ou SIRS, culminando em lesão do endotélio

1. Serviço de Medicina Interna. Hospital Nossa Senhora do Rosário. Centro Hospitalar Barreiro-Montijo. Barreiro. Portugal.

2. Unidade de Cuidados Intensivos. Hospital de Cascais Dr José de Almeida. Cascais. Portugal.

Recebido: 28 de Junho de 2013 - Aceite: 06 de Novembro de 2013 | Copyright @ Ordem dos Médicos 2014 
dos capilares presentes na membrana alvéolo-capilar). A definição utilizada até 2012 era a estabelecida em 1994 na American-European Consensus Conference on ARDS, ${ }^{1}$ caracterizada por: Início agudo; $\mathrm{PaO} 2 / \mathrm{FiO} 2 \leq 200 \mathrm{mmHg}$; Radiografia de Tórax com Infiltrados Bilaterais; e Pressão de Oclusão da Artéria Pulmonar (POAP) $\leq 18 \mathrm{mmHg}$ ou ausência de clínica de hipertensão auricular esquerda. No entanto, na sequência da proposta apresentada por M Ranieri no $24^{\circ}$ Congresso Anual da ESICM em $2011^{2}$ e após Consenso entre representantes da Europa e da América do Norte (The ARDS Definition Task Force), foi publicada uma nova definição em 2012 (The Berlin Definition) ${ }^{3}$ preconizando: 1) início até uma semana após um evento clínico conhecido, ou sintomas respiratórios de novo, ou agravamento de sintomas respiratórios prévios; 2) Radiografia ou Tomografia Computorizada de tórax com opacidades bilaterais, não totalmente explicadas por derrames, colapsos lobares/pulmonares ou nódulos; 3) Insuficiência Respiratória não totalmente explicada por Insuficiência Cardíaca ou sobrecarga hídrica; 4) Classificação do ARDS em ligeiro $(200 \mathrm{mmHg}<\mathrm{PaO} 2 / \mathrm{FiO} 2 \leq 300 \mathrm{mmHg}$ com PEEP ou CPAP $\geq 5 \mathrm{cmH} 2 \mathrm{O}$ ), moderado (100 $\mathrm{mmHg}<\mathrm{PaO} 2 / \mathrm{FiO} 2 \leq 200$ $\mathrm{mmHg}$ com PEEP $\geq 5 \mathrm{cmH} 2 \mathrm{O}$ ) e grave ( $\mathrm{PaO} 2 / \mathrm{FiO} 2 \leq 100$ $\mathrm{mmHg}$ com $\mathrm{PEEP} \geq 5 \mathrm{cmH} 2 \mathrm{O}){ }^{3}$ A significativa incidência (3,5 a 58,7 / 100000 indivíduos/ano, conforme os estudos ${ }^{4}$ e mortalidade (40 a $60 \%)^{4-6}$ justificam estudos adicionais para melhor compreensão desta Síndrome e definição de novas abordagens terapêuticas.

\section{MATERIAL E MÉTODOS}

Este foi um estudo observacional retrospectivo dos casos de ARDS admitidos ou diagnosticados na Unidade de Cuidados Intensivos (UCl) do Hospital de Cascais Dr José de Almeida no período de 20 de Fevereiro de 2010 a 24 de Fevereiro de 2012. Foi estabelecido como princípio de inclusão o cumprimento dos critérios diagnósticos definidos na American-European Consensus Conference on ARDS e como princípio de exclusão a ausência de ventilação mecânica invasiva (VMI). Após aprovação pela
Comissão de Ética, foram pesquisados nos processos clínicos hospitalares (manual e informático) dados demográficos, proveniência, etiologia do ARDS, comorbilidades, Índices de Gravidade (APACHE II e SAPS II nas primeiras $24 \mathrm{~h}$ e mortalidade prevista por SAPS II), SOFA máximo registado e número máximo de disfunções de órgão/ sistema, valor mínimo obtido de ratio $\mathrm{PaO} 2 / \mathrm{FiO} 2$ e de compliance dinâmica (em espirometria), PEEP (positive end-expiratory pressure) e Volume Corrente (VT) máximos aplicados, Pressão de plateau (Pplateau) máxima descrita, dias de VMI, modalidade ventilatória predominantemente utilizada, administração de corticóides segundo protocolo para ARDS (adaptado de Meduri GU et al), ${ }^{7,8}$ terapêuticas de resgate aplicadas [manobras de recrutamento alveolar, decúbito ventral, ventilação oscilatória de alta frequência (HFOV)], complicações, duração do internamento na UCI, óbitos nesse internamento, sobrevida aos 30 dias e aos 2 e 6 meses. Foi calculado o pior Lung Injury Score (LIS) ${ }^{9}$ para cada doente. Para tratamento estatístico dos dados foi utilizado o programa MedCalc - version 12.2.1.0. Foi aplicado o Teste D'Agostino Pearson para Distribuição Normal e Transformação Logarítmica, quando útil, nas variáveis com Coeficiente de Assimetria positivo. Foram utilizados os métodos de Grubbs - double-sided (nível a 0.05), ou de Tukey, ou o Generalized Extreme Studentized Deviate test (nível $\alpha 0,05)$ para identificação de outliers. Foram estabelecidas correlações entre variáveis, consideradas pertinentes, utilizando correlação de Pearson ou correlação de Spearman consoante, respectivamente, tivesse ou não sido aceite normalidade. Foi aplicada Regressão Logística Univariada para relação entre diversas variáveis independentes e as variáveis dependentes 'óbito/não óbito na UCl', 'demora $\leq$ 10 / > 10 dias' e 'VMI $\leq 10$ / > 10 dias'. Para comparação entre amostras foram utilizados: Teste do $t$ de Student para as variáveis contínuas com Distribuição aceite como Normal, Signed rank sum test para as variáveis contínuas sem Distribuição Normal e Teste do Qui quadrado para as variáveis binárias e nominais. Foi considerado significativo $p<0,05$.

Total de oentes admitidos na UCI

20 de Fevereiro de 2010 a 24 Fevereiro de 2012 (2 anos)

$(n=833)$

Doentes cumprindo critério de diagnóstico da

American-European Consensus Conference on ARDS

$(n=41)$

Excluídos os não submetidos a Ventilação

Figura 1 - Diagrama Consort 


\section{RESULTADOS}

Aplicando os critérios de inclusão e exclusão previamentemencionados obteve-se uma amostra de 40 doentes (Fig. 1), dos quais $65 \%$ eram do sexo masculino e $62,5 \%$ apresentavam idade $\geq 65$ anos. A caracterização completa da amostra é exposta nas Tabelas 1 e 2.

Importa salientar que $27,5 \%$ do total de casos de ARDS resultaram de intercorrências/complicações hospitalares.

No que concerne à gravidade do ARDS, é relevante acrescentar que $72,5 \%$ dos doentes apresentaram um

Tabela 1 - Dados demográficos e caracterização do ARDS $(n=40)$

\begin{tabular}{lll}
\hline Variável Valor & Vu
\end{tabular}

Idade (anos)

Mediana (Ampl. Int. P25-P75)

$72,5 \quad(22,00)$

Sexo

Ratio feminino/masculino

$14: 26(\approx 1: 1,86)$

Comorbilidades mais frequentes

Freq. Relativas \% (Freq. Absolutas)

Proveniência

Freq. Relativas \% (Freq. Absolutas)

Índices de Gravidade

Mediana (Amp. Int. P25-P75)

SOFA máximo

Mediana (Amp. Int. P25-P75)

$50,0 \quad(20)$

Diabetes Mellitus $\quad 20,0$

Serviço de Urgência

$47,5 \quad$ (19)

U. Cuidados Intermédios

$15,0 \quad(6)$

Bloco Operatório

$10,0 \quad(4)$

Enfermaria de Cirurgia

$7,5 \quad(3)$

Enfermaria de Medicina

$5,0 \quad(2)$

Enfermaria de Ortopedia

2,5

Outros Hospitais

$12,5 \quad(5)$

APACHE II $1^{\text {as }} 24 \mathrm{~h}(n=38) \dagger$

23,6 $\quad(9,10)$

Média (Desvio Padrão)

$54,5 \quad(27,00)$

11,0

$56,40 \quad(52,400)$

Mortalidade Prevista por SAPS II (\%) $(n=38) \dagger$ Mediana (Ampl. Int. P25-P75)

$\mathbf{N}^{\circ}$ Disfunções Órgão/Sistema

Mediana (Ampl. Int. P25-P75)

Duração do Internamento na UCI (dias)

10,70

Mediana (Ampl. Int. P25-P75)
Pneumonia

52,5

Aspiração de conteúdo gástrico

2,5

Sépsis

37,5

Pancreatite Aguda

5,0

Traumatismo não torácico grave

2,5
Freq. Relativas \% (Freq. Absolutas)

PaO2/FiO2 mínimo (mmHg)

88,0

Média (Desvio Padrão)

Mediana (Ampl. Int. P25-P75) 
Tabela 2 - Abordagens terapêuticas e de ventilação mecânica $(n=40)$

\begin{tabular}{|c|c|c|c|}
\hline \multirow{2}{*}{$\begin{array}{l}\text { Variável } \\
\text { Duração VMI (dias) } \\
\text { Mediana (Ampl. Int. P25-P75) }\end{array}$} & & \multicolumn{2}{|c|}{ Valor } \\
\hline & & 10,0 & $(9,50)$ \\
\hline \multirow{4}{*}{$\begin{array}{l}\text { Modalidade Ventilatória predominante } \\
\text { Freq. Relativas \% (Freq. Absolutas) }\end{array}$} & Pressão Controlada & 87,5 & (35) \\
\hline & Volume Controlado & 5,0 & $(2)$ \\
\hline & PRVC & 5,0 & (2) \\
\hline & Pressão Assistida & 2,5 & (1) \\
\hline $\begin{array}{l}\text { PEEP máxima aplicada }(\mathrm{cmH} 2 \mathrm{O}) \\
\text { Média (Desvio Padrão) }\end{array}$ & & 12,4 & $(4,12)$ \\
\hline $\begin{array}{l}\text { VT máximo aplicado ( } \mathrm{mL} / \mathrm{Kg} \text { peso ideal) } \\
\text { Média (Desvio Padrão) }\end{array}$ & & 8,2 & $(1,34)$ \\
\hline $\begin{array}{l}\text { Pplateau máxima obtida }(\mathrm{cmH} 2 \mathrm{O}) \\
\text { Mediana (Ampl. Int. P25-P75) }\end{array}$ & & 30,5 & $(6,50)$ \\
\hline $\begin{array}{l}\text { Utilização de CT para ARDS } \\
\text { Freq. Relativas \% (Freq. Absolutas) }\end{array}$ & & 47,5 & (19) \\
\hline $\begin{array}{l}\text { Utilização de Relaxante Muscular (Rocurónio) } \\
\text { Freq. Relativas \% (Freq. Absolutas) }\end{array}$ & & 52,5 & (21) \\
\hline $\begin{array}{l}\text { Aplicação de Manobras de Recrutamento Alveolar } \\
\text { Freq. Relativas \% (Freq. Absolutas) }\end{array}$ & & 52,5 & $(21)$ \\
\hline $\begin{array}{l}\text { Aplicação de Decúbito Ventral } \\
\text { Freq. Relativas \% (Freq. Absolutas) }\end{array}$ & & 10,0 & (4) \\
\hline $\begin{array}{l}\text { Utilização de HFOV } \\
\text { Freq. Relativas \% (Freq. Absolutas) }\end{array}$ & & 5,0 & $(2)$ \\
\hline
\end{tabular}

PaO2/FiO2 mínimo $\leq 100 \mathrm{mmHg}$ e que $95 \%$ dos doentes apresentaram um LIS $>2,5$.

Relativamente à Ventilação Mecânica, remete-se para posterior discussão o facto de se ter excedido um VT máximo de $8 \mathrm{~mL} / \mathrm{Kg}$ de peso ideal em $37,5 \%$ dos doentes e uma Pplateau máxima de $30 \mathrm{cmH} 2 \mathrm{O}$ em $50 \%$.

No que diz respeito à terapêutica, o início de corticóides ocorreu numa fase precoce do ARDS ( $\leq 72$ h) em 14 doentes $(73,7 \%$ dos que cumpriram corticoterapia) e numa fase tardia (> 72h) em cinco (26,3\%). Apesar da percentagem de doentes sob corticoterapia precoce que viriam a falecer ser menor do que a dos doentes sob corticoterapia tardia com o mesmo desfecho $(35,7 \%$ vs $60,0 \%)$, essa diferença não apresentou significado estatístico $(p=0,677)$.

Em 32,5\% dos doentes ocorreram complicações, sendo que as mais frequentes foram a Pneumonia Associada à Ventilação em oito doentes (20\%), o Pneumotórax associado à Ventilação/Manobras de Recrutamento Alveolar em cinco (12,5\%) e a Miopatia dos Cuidados Intensivos em 3 (7,5\% do total de doentes). Em todos os doentes com Pneumotórax a PEEP máxima utilizada foi $\geq 10 \mathrm{cmH} 2 \mathrm{O}$ (mediana $=13$, amplitude interquartil P25-P75 = 4) e a Pplateau máxima obtida foi $\geq 28 \mathrm{cmH} 2 \mathrm{O}$ (mediana $=32$, amplitude interquartil P25 - P75 $=5,25$ ). Num desses doentes o VT máximo aplicado foi de apenas $3 \mathrm{~mL} / \mathrm{Kg}$ peso ideal, embora a mediana tenha sido seis (amplitude interquartil P25 - P75 = 1,5). Apesar de não se ter obtido significado estatístico na relação entre 'presença/ausência de complicações' e outcome 'óbito/não óbito na UCl' ( $X^{2} 3,249 ; p=$ 0,071 ), os doentes sem complicações apresentaram uma maior probabilidade de um internamento $\leq 10$ dias (odds ratio 24,556; IC 95\% 2,752 a 219,091; $p=0,004$ ) e de VMI $\leq 10$ dias (odds ratio 54,600; IC 95\% 5,722 a 520,991; $p=$ 0,001) (Tabela 3).

Apenas um doente $(2,5 \%)$ foi traqueostomizado no decurso desse internamento, tendo estado sob VMI durante 38 dias e estando vivo aos 6 meses pós-alta.

A taxa de fatalidade nesse internamento na $\mathrm{UCl}$ foi de $60 \%$ (24 doentes).

No referido período, os doentes com ARDS com necessidade de VMI corresponderam a $4,8 \%$ do total de admissões na $\mathrm{UCl}$ e a $8,1 \%$ dos doentes ventilados. A mediana da duração do internamento - 10,7 dias - foi superior à demora média global na UCI - 6,1 dias - $(p<0,0001)$ e a mortalidade foi muito superior à global: $27,3 \%(p<0,0001)$. No entanto o APACHE II nas primeiras $24 \mathrm{~h}$ não foi significativamente diferente (total de admitidos na UCl: média 21,7; ARDS com VMI: média 23,6; $p=0,1988$ ). 
Tabela 3 - Variáveis independentes estudadas, como eventuais preditoras dos outcomes favoráveis 'Não óbito na UCl', 'VMI $\leq 10$ dias' e 'Demora $\leq 10$ dias'

\begin{tabular}{|c|c|c|c|}
\hline \multirow[b]{2}{*}{ Variáveis independentes } & \multicolumn{3}{|c|}{ 'Não óbito na UCl' } \\
\hline & LR Test & OR & IC $95 \%$ OR \\
\hline Corticoterapia (sim vs não) & $x^{2}=4,924, p=0,026$ & 4,4 & $1,134-17,069$ \\
\hline Idade ( $<65$ vs $\geq 65$ anos) & $X^{2}=11,423, p=0,001$ & 11 & $2,438-49,627$ \\
\hline Sexo (masc vs fem) & $x^{2} 0,891, p=0,345$ & & \\
\hline Etiologia (Pulm. vs Extra-pulm.) & $x^{2} 0,269, p=0,604$ & & \\
\hline PaO2/FiO2 mínimo ( $\leq 100$ vs $>100)$ & $X^{2} 1,320, p=0,251$ & & \\
\hline $\operatorname{LIS}(\leq 2,5$ vs $>2,5)$ & $x^{2} 2,113, p=0,146$ & & \\
\hline PEEP máxima $(<10$ vs $\geq 10 \mathrm{cmH} 2 \mathrm{O})$ & $X^{2} 1,151, p=0,283$ & & \\
\hline$V T$ máximo $(\leq 8 v s>8)$ & $x^{2} 0, p=1,000$ & & \\
\hline Pplateau máxima ( $\leq 30$ vs $>30$ ) & $X^{2} 1,680, p=0,195$ & & \\
\hline $\mathbf{N}^{\circ}$ dias VMI $(\geq 10$ vs $<10)$ & $x^{2} 0,820, p=0,365$ & & \\
\hline M. Recrut. Alveolar (sim vs não) & $X^{2} 2,427, p=0,119$ & & \\
\hline Decúbito Ventral (sim vs não) & $X^{2} 0,440, p=0,507$ & & \\
\hline \multirow[t]{3}{*}{ HFOV (sim vs não) } & $x^{2} 2,113, p=0,146$ & & \\
\hline & \multicolumn{3}{|c|}{ 'VMI $\leq 10$ dias' } \\
\hline & LR Test & OR & IC $95 \%$ OR \\
\hline PEEP máxima $(<10 \mathrm{vs} \geq 10 \mathrm{cmH} 2 \mathrm{O})$ & $x^{2}=6,088, p=0,014$ & 9,714 & $1,081-87,313$ \\
\hline Complicações (não vs sim) & $x^{2}=22,389, p=<0,001$ & 54,600 & $5,722-520,991$ \\
\hline Idade ( $<65$ vs $\geq 65$ anos) & $x^{2} 0,027, p=0,870$ & & $\overline{-}$ \\
\hline Sexo (masc vs fem) & $x^{2} 0,040, p=0,841$ & & \\
\hline Etiologia (Pulm. vs Extra-pulm.) & $X^{2} 0,004, p=0,949$ & & \\
\hline PaO2/FiO2 mínimo ( $\leq 100$ vs $>100)$ & $x^{2} 0,462, p=0,496$ & & \\
\hline LIS $(\leq 2,5$ vs $>2,5)$ & $x^{2} 2,477, p=0,116$ & & \\
\hline$V T$ máximo $(\leq 8 v s>8)$ & $x^{2} 0,027, p=0,870$ & & \\
\hline Pplateau máxima $(\leq 30$ vs $>30)$ & $x^{2} 3,696, p=0,055$ & & \\
\hline Corticoterapia (sim vs não) & $x^{2} 0,123, p=0,726$ & & \\
\hline M. Recrut. Alveolar (sim vs não) & $x^{2} 2,670, p=0,102$ & & 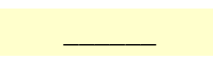 \\
\hline Decúbito Ventral (sim vs não) & $x^{2} 0,045, p=0,833$ & &  \\
\hline \multirow[t]{3}{*}{ HFOV (sim vs não) } & $x^{2} 3,323, p=0,068$ & & \\
\hline & \multicolumn{3}{|c|}{ "Demora $\leq 10$ dias" } \\
\hline & LR Test & OR & IC $95 \%$ OR \\
\hline PEEP máxima (<10 vs $\geq 10 \mathrm{cmH} 2 \mathrm{O}$ ) & $x^{2}=5,192, p=0,023$ & 6,364 & $1,122-36,081$ \\
\hline Complicações (não vs sim) & $x^{2}=14,304, p=<0,001$ & 24,556 & $2,752-219,091$ \\
\hline Idade (<65 vs $\geq 65$ anos) & $x^{2} 0,243, p=0,622$ & & \\
\hline Sexo (masc vs fem) & $x^{2} 0,040, p=0,841$ & & \\
\hline Etiologia (Pulm. vs Extra-pulm.) & $X^{2} 0,496, p=0,481$ & & \\
\hline PaO2/FiO2 mínimo $(\leq 100$ vs $>100)$ & $X^{2} 2,134, p=0,144$ & & \\
\hline LIS $(\leq 2,5$ vs $>2,5)$ & $x^{2} 3,323, p=0,068$ & & \\
\hline VT máximo $(\leq 8$ vs $>8)$ & $X^{2} 0,243, p=0,622$ & & \\
\hline Pplateau máxima $(\leq 30$ vs $>30)$ & $x^{2} 3,696, p=0,055$ & & \\
\hline Corticoterapia (sim vs não) & $x^{2} 0,854, p=0,355$ & & \\
\hline M. Recrut. Alveolar (sim vs não) & $X^{2} 0,854, p=0,355$ & & \\
\hline Decúbito Ventral (sim vs não) & $x^{2} 0,045, p=0,833$ & & $\longrightarrow$ \\
\hline HFOV (sim vs não) & $x^{2} 2,477, p=0,116$ & - & - \\
\hline
\end{tabular}

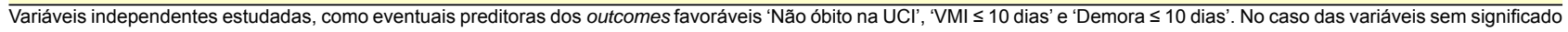
estatístico como preditoras dos outcomes expõe-se apenas o $L R$ test. $[L R$ test = Likelihood ratio test; OR = odds ratio; IC $95 \%=$ Intervalo de Confiança de $95 \%$; masc = masculino; fem = feminino; pulm. = pulmonar; M. Recrut. Alveolar = Manobras de Recrutamento Alveolar] 
Dos sobreviventes nesse internamento, a sobrevida aos 30 dias e aos dois meses foi de $100 \%$. Cinco dos 16 sobreviventes não tinham completado seis meses pós-evento à data da colheita dos dados; um dos 16 doentes faleceu aos quatro meses e 10 estavam vivos aos seis meses.

Foi encontrada correlação positiva entre: PEEP máxima e Dias VMI (Log) - Coeficiente de Correlação $r=0,3189$ ( $p=0,0449$, IC 95\% 0,008181 a 0,5734); LIS e Dias VMI (Log) - Coeficiente de Correlação $r=0,3607$ ( $p=0,0222$, IC $95 \%$ 0,05546 a 0,6043); e Pplateau máxima e $\mathrm{N}^{\circ}$ dias VMI - Spearman's coefficient of rank correlation (rho) $=0,345$ ( $p=0,0294$, IC 95\%: 0,0371 a 0,593 ). Foram testadas, mas sem significado estatístico $(p>0,05)$, as seguintes correlações: PaO2/FiO2 mínimo e Dias VMI (Log); PaO2/FiO2 mínimo e PEEP máxima; Idade e $n^{\circ}$ disfunções órgão/sistema; Idade e $n^{\circ}$ dias $\mathrm{VMI}$; Idade e duração internamento na $\mathrm{UCl}$; SOFA máximo e duração internamento na $\mathrm{UCl}$; SOFA máximo e $\mathrm{n}^{\circ}$ dias $\mathrm{VMI}$.

A probabilidade de outcome favorável 'não óbito na UCl' foi 4,4x (IC 95\% 1,1342 a 17,0693; $p=0,0265$ ) superior nos doentes sob corticoterapia e 11x (IC 95\% 2,4381 a 49,6282; $p=0,0007$ ) superior nos doentes com idade $<65$ anos (Tabela 3). Quando ajustado à idade ( $\geq 65$ anos / $<65$ anos), a probabilidade de outcome favorável foi 6,4x (IC 95\% 1,1179 a 36,$2707 ; p=0,0002$ ) superior nos doentes sob corticoterapia.

Por outro lado, os doentes em quem se aplicou uma PEEP máxima $<10 \mathrm{cmH} 2 \mathrm{O}$ tiveram uma probabilidade 9,7x superior de 'VMI $\leq 10$ dias' (odds ratio 9,714; IC 95\% 1,081 - 87,313; $x^{2} 4,118, p=0,042$ ) e cerca de $6,4 x$ superior de 'demora $\leq 10$ dias' (odds ratio 6,364; IC 95\% 1,122 - 36,081; $x^{2} 4,370, p=0,037$ ). (Tabela 3).

Não se encontrou relação com significado estatístico entre outcome favorável e outras variáveis independentes, conforme explícito na Tabela 3.

\section{DISCUSSÃO E CONCLUSÕES}

Este estudo retrospectivo procura caracterizar os doentes com ARDS admitidos numa Unidade de Cuidados Intensivos de um hospital distrital, cuja área de influência abrange uma população de cerca de 200000 habitantes.

Nesse período de dois anos, como já referido, os doentes com ARDS com necessidade de VMI corresponderam a cerca de $5 \%$ do total de admissões na UCl e cerca de $8 \%$ dos doentes ventilados. De um modo geral, nestes doentes o internamento em Cuidados Intensivos foi mais prolongado e a mortalidade foi muito superior à global nessa $\mathrm{UCl}$.

Esta era uma amostra envelhecida e com uma percentagem elevada de casos de ARDS grave ( $\mathrm{PaO} 2 / \mathrm{FiO} 2$ mínimo $\leq 100 \mathrm{mmHg}$ ), o que poderia justificar a significativa mortalidade verificada (no entanto, dentro do intervalo descrito na bibliografia, de 40 a $60 \%) \cdot{ }^{4-6}$ De facto, a idade < 65 anos associou-se a uma maior probabilidade de sobrevivência ao internamento na $\mathrm{UCI}$. Contudo, não se registou significado estatístico na relação entre $\mathrm{PaO} 2 / \mathrm{FiO} 2$ mínimo $>100 \mathrm{mmHg}$ e outcome favorável.

À semelhança de outros estudos, registou-se um predomínio do sexo masculino $0^{4,10} \mathrm{e}$ as principais etiologias do
ARDS foram a Pneumonia e a Sépsis. ${ }^{4,10,11}$

As comorbilidades mais frequentes poderão, aparentemente, justificar-se pela sua prevalência significativa na população geral, embora fosse necessário outro tipo de estudos para corroborar esta afirmação.

Apesar de se preconizar uma ventilação protectora no ARDS $^{12}$ (com VT máximo de $6-8 \mathrm{~mL} / \mathrm{Kg}$ de peso ideal e Pplateau máxima de $30 \mathrm{cmH} 2 \mathrm{O}$ ) nem sempre esta permite obter trocas gasosas minimamente adequadas, pelo que houve necessidade de exceder os limites numa percentagem significativa dos doentes da amostra. No entanto, nesses indivíduos não houve associação, com significado estatístico, a pior outcome.

Nesta amostra, cerca de metade dos doentes cumpriram corticoterapia segundo protocolo para ARDS. ${ }^{7,8}$ Dado que se trata de um estudo retrospectivo, não foi possível apurar em todos os casos a justificação ou critério para o início de corticóide, assumindo-se que se baseou na gravidade do quadro e no senso clínico do(s) médico(s).

Até à data, diversos estudos publicados concluíram não ser benéfica a administração, precoce ou tardia, de corticóides no ARDS. Contrariamente, Meduri et al ${ }^{7,8}$ obtiveram uma redução significativa da mortalidade em $\mathrm{UCl}$, particularmente mediante a sua utilização precoce em doentes com ARDS grave.

Na nossa amostra, caracterizada por uma elevada percentagem de casos graves, o uso de corticóides associou-se a uma maior probabilidade de sobrevivência nesse internamento na UCI. Não teve, no entanto, influência com significado estatístico sobre a duração da VMI e a demora na UCI.

Este estudo tem como principal limitação o facto de ser observacional retrospectivo. Adicionalmente, algumas variáveis foram difíceis de analisar devido à ausência de registos padronizados. Além disso, apesar da maioria dos doentes ter cumprido corticoterapia precocemente, foram igualmente incluídos neste estudo, indistintamente, aqueles que a iniciaram tardiamente. Pelo reduzido número de doentes submetidos a Decúbito Ventral e a HFOV, o estudo não permite retirar conclusões válidas acerca destes dois tipos de terapêutica de resgate.

No entanto, a escassez de publicações sobre ARDS a nível nacional motivou o empenho neste estudo.

Os autores concluem que o ARDS se associa a internamentos prolongados em UCl (deduzindo-se maiores custos inerentes) e a significativa mortalidade. As dúvidas aqui suscitadas justificam a elaboração de estudos prospectivos futuros dedicados a confirmar o benefício aparente da corticoterapia no ARDS e a identificar o/(s) subgrupo/(s) de doentes que mais justificam a sua utilização.

\section{CONFLITOS DE INTERESSE}

Os autores declararam a inexistência de conflitos de interesse.

\section{FONTES DE FINANCIAMENTO}

Os autores declaram que não houve qualquer tipo de apoio financeiro para este trabalho. 


\section{REFERÊNCIAS}

1. Bernard GR, Artigas A, Brigham KL, Carlet J, Falke K, Hudson L, et al. The American-European Consensus Conference on ARDS - definitions, mechanisms, relevant outcomes, and clinical trial coordination. Am J Respir Crit Care Med. 1994;149: 818-24.

2. Ranieri M. Presentation on ARDS. [Consultado 2012 Fev 24]. Disponível em http://www.esicm.org/07-congresses/0A-annual-congress/webTv_ranieri.asp.

3. The ARDS Definition Task Force. Acute respiratory distress syndrome, the Berlin definition. JAMA. 2012;307: 2526-33.

4. Villar J, Blanco J, Añón JM, Santos-Bouza A, Blanch L, Ambrós A, et al. The ALIEN study: incidence and outcome of acute respiratory distress syndrome in the era of lung protective ventilation. Intensive Care Med. 2011;37:1932-41.

5. Zambon M, Vincent JL. Mortality rates for patients with acute lung injury/ ARDS have decreased over time. Chest. 2008;133:1120-7.

6. Ware LB, Matthay MA. The acute respiratory distress syndrome. N Engl J Med. 2000;342:1334-49.

7. Meduri GU, Golden E, Freire AX, Taylor E, Zaman M, Carson SJ, et al. Methylprednisolone infusion in early severe ARDS - results of a randomized controlled trial. Chest. 2007;131:954-63.

8. Meduri GU, Headley AS, Golden E, Carson SJ, Umberger RA, Kelso $\mathrm{T}$, et al. Effect of prolonged methylprednisolone therapy in unresolving acute respiratory distress syndrome: a randomized controlled trial. JAMA. 1998;280:159-65.

9. Raghavendran K, Napolitano LM. ALI and ARDS: Challenges and advances. Crit Care Clin. 2011;27:429-37.

10. Agarwal $R$, Srinivas $R$, Nath $A$, Jindal SK. Is the mortality higher in the pulmonary vs the extrapulmonary ARDS?-A metaanalysis. Chest. 2008;133:1463-73.

11. Sheu CC, Gong MN, Zhai R, Chen F, Bajwa EK, Clardy PF, et al. Clinical
Characteristics and Outcomes of Sepsis-Related vs Non-Sepsis-Related ARDS. Chest. 2010;138: 559-67.

12. NIH NHLBI ARDS Clinical Network Mechanical Ventilation Protoco Summary. [Consultado 2012 Fev 24]. Disponível em http://www.ardsnet. org/system/files/Ventilator\%20Protocol\%20Card.pdf.

13. Antonelli M, Bonten M, Chastre J, Citerio G, Conti G, Curtis JR, et al. Year in review in Intensive Care Medicine 2011: III. ARDS and ECMO, weaning, mechanical ventilation, noninvasive ventilation, pediatrics and miscellanea. Intensive Care Med. 2012;38:542-56.

14. Diaz JV, Brower R, Calfee CS, Matthay MA. Therapeutic strategies for severe acute lung injury. Crit Care Med. 2010;38:1644-50.

15. Artigas A, Bernard GR, Carlet J, Dreyfuss D, Gattinoni L, Hudson L, et al. The American-European Consensus Conference on ARDS, Part 2 - Ventilatory, pharmacologic, supportive therapy, study design strategies, and issues related to recovery and remodeling. Acute respiratory distress syndrome. Am J Respir Crit Care Med. 1998;157:1332-47.

16. Laycock $\mathrm{H}$, Rajah $\mathrm{A}$. Acute lung injury and acute respiratory distress syndrome: A review article. BJMP. 2010;3:324.

17. Girard TD, Bernard GR. Mechanical ventilation in ARDS A state-of-theart review. Chest. 2007;131: 921-9.

18. Plantados CA, Schwartz DA. The acute respiratory distress syndrome. Ann Intern Med. 2004;141:460-70.

19. Charron C, Repesse X, Bouferrache K, Bodson L, Castro S, Page B, et al. $\mathrm{PaCO}_{2}$ and alveolar dead space are more relevant than $\mathrm{PaO}_{2} / \mathrm{FiO}_{2}$ ratio in monitoring the respiratory response to prone position in ARDS patients: a physiological study. Critical Care. 2011;15:R175.

20. Liu LL, Aldrich JM, Shimabukuro DW, Sullivan KR, Taylor JM, Thornton $\mathrm{KC}$, et al. Rescue therapies for acute hypoxemic respiratory failure. Anesth Analg. 2010;11:693-702. 


\section{Síndrome de Dificuldade Respiratória Aguda: Casuística de Dois Anos numa Unidade de Cuidados Intensivos \\ Acta Med Port 2014:27:211-217}

Publicado pela Acta Médica Portuguesa, a Revista Científica da Ordem dos Médicos

Av. Almirante Gago Coutinho, 151

1749-084 Lisboa, Portugal.

Tel: +351 218428215

E-mail: submissao@actamedicaportuguesa.com

www.actamedicaportuguesa.com

ISSN:0870-399X | e-ISSN: 1646-0758

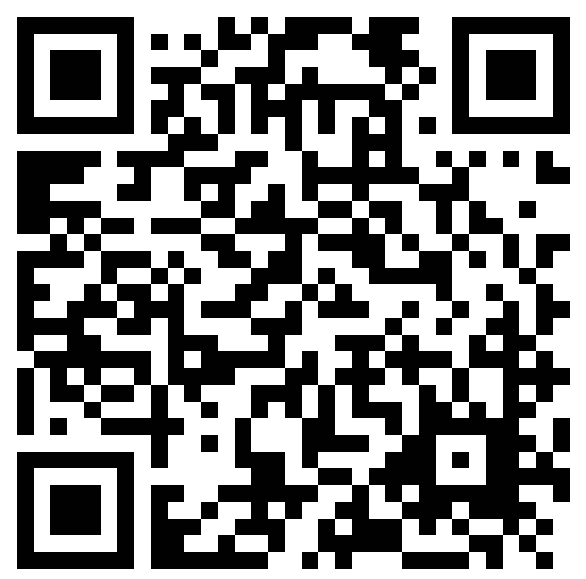

